\title{
CORRIGENDUM
}

doi:10.1038/nature08846

\section{The sequence and de novo assembly of the giant panda genome}

Ruiqiang Li, Wei Fan, Geng Tian, Hongmei Zhu, Lin He, Jing Cai, Quanfei Huang, Qingle Cai, Bo Li, Yinqi Bai, Zhihe Zhang,

Yaping Zhang, Wen Wang, Jun Li, Fuwen Wei, Heng Li, Min Jian, Jianwen Li, Zhaolei Zhang, Rasmus Nielsen, Dawei Li, Wanjun Gu, Zhentao Yang, Zhaoling Xuan, Oliver A. Ryder,

Frederick Chi-Ching Leung, Yan Zhou, Jianjun Cao, Xiao Sun, Yonggui Fu, Xiaodong Fang, Xiaosen Guo, Bo Wang, Rong Hou, Fujun Shen, Bo Mu, Peixiang Ni, Runmao Lin, Wubin Qian,

Guodong Wang, Chang Yu, Wenhui Nie, Jinhuan Wang,

Zhigang Wu, Huiqing Liang, Jiumeng Min, Qi Wu, Shifeng Cheng, Jue Ruan, Mingwei Wang, Zhongbin Shi, Ming Wen, Binghang Liu, Xiaoli Ren, Huisong Zheng, Dong Dong, Kathleen Cook, Gao Shan, Hao Zhang, Carolin Kosiol, Xueying Xie, Zuhong Lu,

Hancheng Zheng, Yingrui Li, Cynthia C. Steiner,

Tommy Tsan-Yuk Lam, Siyuan Lin, Qinghui Zhang, Guoqing Li,

Jing Tian, Timing Gong, Hongde Liu, Dejin Zhang, Lin Fang, Chen Ye, Juanbin Zhang, Wenbo Hu, Anlong Xu, Yuanyuan Ren, Guojie Zhang, Michael W. Bruford, Qibin Li, Lijia Ma, Yiran Guo, Na An, Yujie Hu, Yang Zheng, Yongyong Shi, Zhiqiang Li, Qing Liu, Yanling Chen, Jing Zhao, Ning Qu, Shancen Zhao, Feng Tian, Xiaoling Wang, Haiyin Wang, Lizhi Xu, Xiao Liu, Tomas Vinar, Yajun Wang, Tak-Wah Lam, Siu-Ming Yiu, Shiping Liu, Hemin Zhang, Desheng Li, Yan Huang, Xia Wang, Guohua Yang, Zhi Jiang, Junyi Wang, Nan Qin, Li Li, Jingxiang Li, Lars Bolund, Karsten Kristiansen, Gane Ka-Shu Wong, Maynard Olson, Xiuqing Zhang, Songgang Li, Huanming Yang, Jian Wang \& Jun Wang

Nature 463, 311-317 (2010)

In this Article, the Latin species name of the giant panda was written incorrectly as Ailuropoda melanoleura. The correct name is Ailuropoda melanoleuca. 